\title{
El dominio de lo mental en la filosofía de Williamson*
}

\author{
(The Domain of the Mental in Williamson's Philosophy)
}

\author{
Manuel Pérez Otero
}

Recibido: 07/02/2017

Versión final: 16/09/2017

BIBLID 0495-4548(2018)33:1p.23-40

DOI: $10.1387 /$ theoria. 17629

RESUMEN: Según Williamson, saber y creer son estados mentales, pero creer algo verdadero y creer justificadamente algo verdadero no lo son. Ese tratamiento discriminatorio es relevante para la epistemología de Williamson. Su principal tesis epistemológica negativa (sobre la supuesta imposibilidad de definir el saber conforme a cierto formato tradicional) y su principal tesis epistemológica positiva (una definición, alternativa, del saber) están en peligro si su teoría metafísica sobre lo mental es incorrecta. Presento aquí un problema para dicha teoría: impone limitaciones implausibles a los posibles usos de conceptos y expresiones lingüísticas. Describiré algunas opciones que tendría Williamson para evitar el problema; pero sostendré que acarrean cierta dosis de arbitrariedad.

Palabras clave: definiciones del conocimiento; creencia verdadera; conceptos mentales; estados factivos; conceptos conjuntivos.

ABSTRACT: For Williamson, knowing and believing are mental states, but believing truly and justifiedly-and-truly believing are non-mental states. This discriminatory approach is relevant to his epistemology: his main negative epistemological thesis (on the alleged impossibility of defining knowledge in accordance with a traditional scheme) and his main positive epistemological thesis (his own alternative definition of knowledge) depend on his metaphysical theory about the demarcation of the mental. I present here a problem for Williamson's theory of the mental: it imposes implausible restrictions on possible uses of concepts and linguistic expressions. I will describe some options that Williamson would have at his disposal to evade the problem; but I maintain that these options carry some degree of arbitrariness.

Keywords: definitions of knowledge; true belief; mental concepts; factive states; conjunctive concepts.

* Algunas de las ideas desarrolladas en este artículo se han presentado en el IV Congreso de la Asociación Latinoamericana de Filosofía Analítica (S. José de Costa Rica, Mayo 2016) y en el VIII Congreso de la Sociedad Española de Filosofía Analítica (Oviedo, Noviembre 2016; cf. Pérez Otero 2016b). Agradezco a las respectivas audiencias, y a otras personas con quienes he discutido sobre estos contenidos, sus comentarios, críticas y sugerencias. Estoy en deuda, especialmente, con Juan Comesaña, José Díez, Manuel García-Carpintero, Manolo Martínez, Daniel Quesada, Sven Rosenkranz y Ernesto Sosa. Financiación: Proyecto «Objetividad-subjetividad en el conocimiento y en la representación singular» (FFI2015-63892-P), (MINECO, AEI/FEDER, UE). Proyecto «Perspectival Thoughts and Facts: New Questions» (FFI2016-81858-REDC; Red CONSOLIDER 2016, Acción de Dinamización, Red de Excelencia) / Grupo de investigación consolidado LOGOS (2017SGR63), AGAUR. 


\section{Introducción}

Imaginemos que $\mathrm{P}$ es una determinada proposición cotidiana contingente sobre el entorno externo. La propiedad, o el estado, o la condición, de creer que $\mathrm{P}$ es un ejemplo paradigmático de estado mental. Respecto a otros supuestos estados mentales, la cuestión no sería tan nítida. En su influyente libro Knowledge and its Limits, Williamson afirma que también saber que $\mathrm{P}$ es un estado mental. Por otra parte, rechaza que sean estados mentales tener la creencia verdadera de que $\mathrm{P}$ y tener justificadamente la creencia verdadera de que $P$.

Ese tratamiento discriminatorio entre los antedichos cuatro presuntos tipos de estados mentales (creer, saber, creer algo verdadero, creer justificadamente algo verdadero) en su teoría sobre la demarcación de lo mental desempeña un papel crucial en varias de sus propuestas epistemológicas relativas a las posibilidades de definir el conocimiento. Sobre tales posibilidades, Williamson defiende diversas tesis negativas y una tesis positiva. Conforme a una de las tesis negativas, es imposible definir el conocimiento usando cierto formato tradicional específico (de carácter conjuntivo). La tesis positiva es su propia definición del saber.

Hay tensiones internas en la teoría sobre lo mental de Williamson, que conducirían a sostener - contra sus propósitos- que si saber es un (tipo de) estado mental entonces no hay buenas razones para afirmar que no lo sean creer algo verdadero y creer justificadamente algo verdadero. Si eso es correcto, su demarcación del ámbito mental sería errónea, o al menos insuficientemente motivada. Estos inconvenientes repercuten negativamente en sus tesis epistemológicas: tanto la tesis negativa mencionada (sobre la imposibilidad de definir el saber conforme a cierto formato tradicional-conjuntivo) como la tesis positiva (su definición del saber) están en riesgo.

Expondré esos inconvenientes, así como las opciones que tendría Williamson para replicar. Mi posición se basará, principalmente, en una hipótesis metafísica sobre posibles usos de ciertos conceptos que - según defenderé- son de carácter mental si el concepto de saber es mental. Williamson debería negar que tal hipótesis sea posible, o bien negar que los conceptos involucrados sean mentales. Sostendré que esas opciones, aunque no sean muy claramente implausibles, comportan cierta dosis de arbitrariedad.

Este artículo puede contemplarse como una crítica a la posición de Williamson. Ésa es mi perspectiva prioritaria. No obstante, quien crea que las opciones posibles de réplica por parte de Williamson (que describiré) son razonables, puede contemplar mi tarea como - al menos - una elucidación de qué costes teóricos supondría para Williamson librarse de las objeciones aquí presentadas (elucidación que no se encuentra - por lo que yo sé- en ningún otro trabajo publicado que aborde estos temas). Dada esa dialéctica general, creo que la discusión global tiene un interés intrínseco para lectores que adopten cualquiera de estos tres posicionamientos teóricos (y, por supuesto, para muchos lectores que suspendan el juicio): (i) aceptar mis críticas y considerar que la teoría de Williamson tiene graves inconvenientes; (ii) considerar que vale la pena abonar los costes teóricos que le supondrían a Williamson zafarse de tales inconvenientes tomando algunas de esas opciones de réplica; (iii) postular alguna réplica concreta a mis críticas distinta a las que aquí identificaré. 


\section{Estados mentales y conceptos mentales}

Williamson asume una distinción relativamente bien establecida entre, por una parte, conceptos y, por otra, estados (o condiciones, o propiedades). ${ }^{1}$ Uno de los ejemplos con que ilustra esa distinción concierne al oro. Hay diferentes conceptos que corresponden a un cierto estado o propiedad: la propiedad de ser oro. Dos de esos conceptos son el concepto expresado por 'oro' y el concepto expresado por 'elemento químico con número atómico 79'. El estado, ser oro, no es idéntico a ninguno de tales conceptos; además, esos dos conceptos también son diferentes uno del otro.

Usando una notación que es frecuente en la literatura, emplearé mayúsculas para referirme a conceptos (Williamson usa, a veces, letra cursiva con el mismo propósito, como constataremos en la próxima cita de su libro, al final de esta sección). Así, los dos conceptos mencionado son el concepto ORO y el concepto ELEMENTO QUÍMICO CON NÚMERO ATÓMICO 79. Al hablar de la relación entre un concepto y el estado al que corresponde, Williamson dice a veces - y aquí seguiré su práctica- que el concepto (por ejemplo ORO) es un concepto de ese estado (del estado de ser oro, en ese caso). En alguna ocasión, emplea la noción de concepto de forma laxa, o algo diferente, indicando que los conceptos son representaciones lingüísticas o mentales (cf. Williamson 2009a, nota 1). Conforme a ese uso, también puede decirse que el concepto refiere al estado correspondiente, el estado del cual es un concepto. Las cuestiones sobre los estados son de orden metafísico; las cuestiones sobre los conceptos son de orden epistémico-representacional. En resumidas cuentas, las siguientes aseveraciones ilustran esas diversas opciones notacionales y terminológicas: el concepto expresado por 'oro' es ORO; es un concepto del estado, o la propiedad, de ser oro; su referencia es ese estado. Otros posibles conceptos tendrán la misma referencia, por ejemplo el concepto ELEMENTO QUÍMICO CON NÚMERO ATÓMICO 79.

Como es obvio, esas distinciones se aplican también al concepto epistemológico principal, el concepto SABER, y otros conceptos con él emparentados, como CREER. El estado de saber difiere del concepto SABER. Además, otros conceptos diferentes al concepto expresado por 'saber' también tendrán como referencia ese estado. Pasa lo mismo respecto a CREER, CREER ALGO VERDADERO, CREER JUSTIFICADAMENTE ALGO VERDADERO, etc.

Con frecuencia, Williamson desarrolla la discusión hablando indistintamente de saber o de saber que P, relativamente a una cierta proposición P; análogamente respecto a creer y otros presuntos estados mentales. Esa fluctuación no genera ninguna confusión. También en esto seguiré sus usos (ya lo he hecho así en la introducción).

Interesa hacer otra aclaración. Creer algo verdadero [truly believe] no es el estado, o propiedad, monádico consistente en tener alguna creencia verdadera. Debe interpretarse análogamente al estado general de creer: como el estado, o propiedad, diádico que tiene un sujeto relativamente a proposiciones que cree y que son verdaderas (es decir, tampoco creer es la propiedad monádica de tener alguna creencia). Pasa lo mismo respecto a creer justificadamente algo verdadero. En parte para que no haya confusión respecto a ese punto y en parte por razones de simplicidad - para facilitar la exposición - voy a utilizar una única palabra para referirme al estado de creer algo verdadero, y también una única palabra para

1 La diferencia que establece Williamson entre estados y condiciones es irrelevante para nuestra discusión (cf. Williamson 2009b, 330). Hablaré indistintamente de estados, condiciones o propiedades. 
referirme al estado de creer justificadamente algo verdadero. Digamos que, por estipulación, doxacertar que $\mathrm{P}$ es tener la creencia verdadera de que $\mathrm{P}$ y justacertar que $\mathrm{P}$ es tener la creencia verdadera y justificada de que P. Empleando esa terminología, reformulemos el contraste propuesto por Williamson: creer y saber son estados mentales; doxacertar y justacertar no lo son. $\mathrm{O}$, de forma más específica: si $\mathrm{P}$ es una determinada proposición cotidiana contingente sobre el entorno externo, creer que $\mathrm{P}$ y saber que $\mathrm{P}$ son estados mentales; doxacertar que $\mathrm{P}$ y justacertar que $\mathrm{P}$ no lo son.

Naturalmente, incurriría en un error quien creyera que por acunar estipulativamente un único término para referirnos a esos estados (doxacertar y justacertar) se avanza algo en favor de aceptar que son suficientemente similares a creer y saber, y por tanto -en principio- también deberían considerarse estados de carácter mental. Sigue abierta la cuestión de si son o no estados mentales. Pero también está abierta la cuestión de si saber lo es. En la sección 4 defenderé que - siguiendo los criterios del propio Williamson-si saber es un estado mental, entonces es razonable aceptar que doxacertar y justacertar también son estados mentales. Esa defensa no dependerá de una asignación estipulativa de significado como la que aquí se ha realizado.

Las condiciones de identidad de los conceptos son —como cabe suponer- más finas que las de los estados. Según Williamson:

(W1) $\mathrm{E}_{1}$ y $\mathrm{E}_{2}$ son el mismo estado si y sólo si necesariamente ( $x$ está en $\mathrm{E}_{1}$ syss $x$ está en $\mathrm{E}_{2}$ )

(W2) SER C y [SER C Y TAL QUE T] son conceptos diferentes, incluso cuando son lógicamente equivalentes (por ejemplo, si ' $T$ ' es una verdad lógica trivial)

Veremos a continuación algunas otras tesis sustantivas pertenecientes a la teoría de Williamson sobre el dominio de lo mental, es decir su teoría sobre los estados y los conceptos mentales, y sobre las relaciones entre ellos. Llamaré TWM (por «teoría de Williamson sobre lo mental») a dicha teoría. (Cf., en especial, los capítulos 1 y 2 de Williamson 2000.) Forman parte de TWM las afirmaciones (W1) y (W2), así como cuatro tesis que he enunciado previamente: creer es un estado mental; saber es un estado mental; doxacertar no es un estado mental; justacertar no es un estado mental.

¿Qué determina el carácter mental o no mental de un estado? ¿Cómo se vincula dicho carácter con el carácter mental o no mental de los conceptos que refieren a ese estado? Williamson proporciona una respuesta precisa a la segunda pregunta, que permite avanzar también en la clarificación de la primera. Su TWM incluye esta tesis:

(W3) El estado E es mental si y sólo podría haber un concepto mental de E

Que cierto estado sea mental no implica que todo concepto de dicho estado (es decir, que refiera a dicho estado) sea mental. Como (W3) sugiere, unos lo serán y otros no. En concreto, Williamson clasifica como mental el concepto SABER; ésa es otra tesis crucial de TWM. Por otro lado, un paradigma de concepto no mental es SER VERDADERO. Y también se supone que el estado de ser verdadero es no mental (es decir, dado (W3), ningún posible concepto mental tendrá ese estado como referencia). ${ }^{2}$

2 Advirtamos que, según (W3), la mera posibilidad de que existiera un concepto con las características indicadas (que fuera mental y fuera un concepto de E) ya determina que $\mathrm{E} e s$ un estado mental. (Si aten- 
La distinción entre el estado de saber y el concepto SABER resulta a menudo irrelevante. Quizá por ello Williamson enfatiza sobre todo el carácter mental del estado, cuando en realidad - a la luz de (W3) - parece que, al menos en el orden epistémico, sería el carácter mental del concepto SABER lo prioritario, habida cuenta de que el estado será también referencia de otros conceptos no mentales, con lo cual quedaría abierta la posibilidad de no ser un estado mental, si resultara que ninguno de ellos lo fuera. Aparentemente, debemos aceptar que saber es un estado mental porque SABER es un concepto mental.

Conviene prestar atención a esta otra tesis importante de TWM:

(W4) Si el concepto $\mathrm{C}$ es una conjunción de los conceptos $\mathrm{C}_{1}, \ldots \mathrm{C}_{\mathrm{n}}$, y uno de tales conceptos, $\mathrm{C}_{\mathrm{i}}$, no es un concepto mental, entonces $\mathrm{C}$ no es un concepto mental.

La proposición (W4) es clave para sustentar el contraste que hemos señalado desde el inicio, entre el carácter mental de ciertos estados (creer; saber) y el carácter no mental de otros (doxacertar; justacertar). Williamson asume que el concepto DOXACERTAR QUE P (es decir, el concepto TIENE LA CREENCIA VERDADERA DE QUE P, pues ése es el concepto expresado por 'doxacertar que P', dado el modo en que hemos introducido este verbo) y el concepto conjuntivo [TIENE LA CREENCIA DE QUE P] Y [P ES VER$\mathrm{DAD}$ ] son el mismo concepto. No tengo ninguna objeción a dicho supuesto. Siendo así, (W4) le permite concluir que el concepto DOXACERTAR QUE P (o, en términos generales, el concepto DOXACERTAR) no es mental. De forma análoga se concluiría que JUSTACERTAR tampoco es un concepto mental. Por el contrario, según Williamson, SABER sí es un concepto mental; no es un concepto híbrido, compuesto conjuntivamente de algo mental y algo no mental. No podría invocarse la tesis (W4) para refutar el carácter mental de SABER.

Aunque esos dos conceptos híbridos (DOXACERTAR y JUSTACERTAR, que combinan, conjuntivamente, un concepto mental con otro no mental) no sean mentales, sus correspondientes estados podrían ser mentales, conforme a (W3): serían estados mentales si fueran también la referencia de otros posibles conceptos que sí fueran mentales. Pero, por lo que respecta a doxacertar (y su posición sería análoga respecto a justacertar) Williamson rechaza esa posibilidad, dando a entender que se trataría de una coincidencia difícilmente explicable y, por tanto, sería sensato suponer que no es el caso:

Si el concepto cree con verdad [believes truly; es decir, el concepto DOXACERTAR] es no mental, su imaginada coextensividad con un concepto mental sería una coincidencia metafísica extraña [bizarre]. (Williamson 2000, 29)

Por tanto, cabe atribuir a Williamson esta otra tesis:

(W5) Es razonable suponer que un concepto mental no será necesariamente coextensional con un concepto no mental.

diéramos a otros aspectos de la filosofía de Williamson, esta observación ni siquiera sería necesaria, pues cabe contemplar los conceptos desde un punto de vista abstracto, conforme al cual todo concepto posible es un concepto real.) 


\section{Definiciones tradicional-conjuntivas del saber}

Con objeto de formular una de las tesis negativas de Williamson sobre la indefinibilidad del saber, presentemos primero dos caracterizaciones. Una definición es conjuntiva si su definiens es un concepto conjuntivo, entendiendo por tal un concepto con la forma correspondiente a $C_{1} Y C_{2} Y \ldots C_{n}($ para $\mathrm{n}>1)$. Una definición tradicional-conjuntiva del saber es una definición conjuntiva y no circular del saber entre cuyos conjuntos se incluye el concepto P ES VERDADERO. Así, por ejemplo, el clásico análisis tripartito del conocimiento refutado - según la opinión mayoritaria, que comparto- por los casos Gettier es una definición tradicional-conjuntiva. ${ }^{3}$

Entre otras varias afirmaciones pesimistas sobre las posibilidades de definir el conocimiento, Williamson sostiene esta proposición:

(W6) Ninguna definición tradicional-conjuntiva proporcionará condiciones necesarias y suficientes correctas para que haya conocimiento.

Ésta es otra manera de expresar (W6): No es necesariamente verdadera ninguna definición no circular del conocimiento que tenga el formato de $\ll \mathrm{S}$ sabe que $\mathrm{P}$ si y sólo si [ $\mathrm{P}$ es verdadero, $\left.\mathrm{C}_{1} \mathrm{y} \ldots \mathrm{C}_{\mathrm{n}}\right] \gg($ para $\mathrm{n}>0)$.

¿Qué justificación ofrece Williamson para afirmar (W6)? Presenta consideraciones de dos tipos. En primer lugar, aduce una argumentación inductiva simple: a su juicio, se han encontrado contraejemplos a todas las definiciones tradicional-conjuntivas del saber presentadas hasta ahora. Si eso es verdad, proporcionaría cierta justificación para suponer que cualquier otra definición de ese tenor que se presente correrá la misma suerte. (Cf. Williamson 2000, cap. 1.) No abordaré aquí esa cuestión, que nos alejaría de la discusión principal. Pero conviene tener en cuenta una complicación: la controversia sobre la supuesta «base empírica» que sustentaría la generalización (haber «observado», presuntamente, el fracaso de tales definiciones tradicional-conjuntivas previas) deriva, también, de que es poco claro su alcance, pues dista de ser obvio si una determinada definición es o no conjuntiva. El asunto depende de hipótesis sobre cuál sea la forma genuina de una definición, que puede no corresponder trivialmente con su forma superficial. ${ }^{4}$

En segundo lugar, algunas otras partes de su teoría TWM confieren apoyo a (W6). Concretamente, sus tesis de que SABER es un concepto mental y SER VERDADERO no lo es, combinadas con las tesis (W4) y (W5) hacen razonable sostener (W6). Si SER VERDADERO no es un concepto mental, entonces el definiens de una definición tradicionalconjuntiva expresa un concepto que - en virtud de (W4) - tampoco es mental. Por tanto, si el definiendum y el definiens de una definición tradicional-conjuntiva fueran necesariamente coextensionales (es decir, si (W6) fuera falsa), tendría lugar la extraña, o difícilmente explicable, coincidencia metafísica que (W5) desestima por considerarla poco razonable. Con terminología algo diferente, Williamson destaca ese resultado, cuando sugiere que su teoría, TWM, no implica estrictamente pero recomienda aceptar (W6):

3 Dicho análisis establece lo siguiente: $S$ sabe que $P$ si y sólo si ( $S$ cree que $P, P$ es verdadero y $S$ está justificado en creer que P). Cf. Gettier (1963).

4 Por ejemplo, no son coincidentes las opiniones de Williamson y Sosa respecto a cuándo una definición estaría ejemplificando o no el formato desestimado por la tesis (W6). Cf. Sosa $(2009,206 ; 2015,15-$ 16) y Williamson (2000, cap. 1; 2009b, 364). 
Si un concepto no mental fuera necesariamente coextensional con el concepto mental sabe, serían conceptos del mismo estado mental. La explicación presente [la teoría que he llamado $T W M]$ no implica estrictamente que ningún análisis del tipo tradicional proporciona condiciones necesarias y suficientes correctas para el conocimiento [no implica estrictamente (W6)]. Pero una vez aceptamos que que el concepto sabe no es un concepto complejo del tipo tradicionalmente concebido [una vez aceptamos que SABER, siendo un concepto mental, no es - en virtud de (W4) - idéntico a un concepto con la forma correspondiente a $\mathrm{C}_{1} \mathrm{Y} \mathrm{C}_{2} \mathrm{Y} \ldots \mathrm{C}_{\mathrm{n}}$ ], qué razón tenemos para esperar que algún concepto complejo tal proporcione siquiera condiciones necesarias y suficientes correctas para el conocimiento? (Williamson 2000, 30. Las acotaciones clarificatorias entre corchetes son mías.).

Sin embargo, en la sección 4 intentaré justificar que — dados esos otros supuestos de TWM - la tesis (W5) no resulta creíble. Argumentaré que si SABER es un concepto mental, entonces hay posibles conceptos mentales necesariamente coextensionales con DOXACERTAR y JUSTACERTAR, conceptos estos últimos que — según TWM - no son mentales; con lo cual, (W5) estará en conflicto. (En las secciones 6 y 7 describiré los costes que supondría para Williamson rechazar esa argumentación.) Si mi posición es acertada, se disipan los indicios no meramente inductivos aportados por Williamson en favor de su tesis negativa (W6), pues dichos indicios dependen crucialmente de (W5).

\section{La definición de Williamson del saber}

En la sección 1.4 de Williamson (2000) se defiende una definición del conocimiento. Williamson la presenta en dos fases: primero expone una versión menos precisa, pero ya suficientemente clara (p. 34); después articula con mayor detalle lo que en un texto posterior llama la explicación completa [the full account] (pp. 37-41; cf. Williamson 2009b, 364). Desarrollaré la discusión concentrándome en la definición inicial (en esta sección), porque los puntos relevantes no dependen de la diferencia entre ambas. Pero en la sección 5 también mencionaré y comentaré su definición precisa oficial.

Ésta es la definición propuesta por Williamson:

(D) Saber es el (tipo de) estado mental factivo más general

Clarifiquemos qué significa eso. La factividad del saber consiste en que cualquier oración con la forma de $\ll S$ sabe que $P »$ implica la verdad de la cláusula subordinada, implica que 'P' es verdad. ${ }^{5}$ Hay otros estados mentales factivos, como por ejemplo recordar. Que saber sea el más general de todos ellos quiere decir lo siguiente: si el verbo ' $F$ ' denota un estado mental de actitud proposicional y es también un verbo factivo, entonces saber es tan general o más que dicho estado en el sentido de que toda oración con la forma de «S F que $\mathrm{P} \gg \mathrm{im}$ plicará $\ll S$ sabe que $P »$.

Constatemos que su TWM es crucial para que la definición (D) pueda tener siquiera alguna plausibilidad. Como es obvio, doxacertar y justacertar son estados factivos, y son más generales que saber (saber implica doxacertar y también implica justacertar, pero no al contrario). Williamson acepta eso. Pero cree que ello no refuta su definición, pues consi-

5 En Pérez Otero (2017, sección 3) he criticado la opinión — muy minoritaria- de que saber no es un estado factivo. 
dera que doxacertar y justacertar no son estados mentales. Por tanto, (D) requiere - entre otras cosas - la verdad de tres tesis concretas de TWM: saber es un estado mental; doxacertar no es un estado mental; justacertar no es un estado mental.

Su línea de demarcación de lo mental desempeña, pues, un papel esencial para la defensa de la principal tesis positiva respecto al saber sostenida por Williamson: su definición (D). En la sección previa hemos visto que también es esencial para la defensa de su tesis negativa (W6). Pero de la discusión en la próxima sección podremos inferir que esa posición sobre qué delimita lo mental resulta inestable.

\section{Una hipótesis metafísico-antropológica sobre usos de ciertos conceptos}

Recordemos algunos datos relevantes concernientes a por qué — según Williamson-SABER es un concepto mental aunque DOXACERTAR y JUSTACERTAR no sean conceptos mentales (en las secciones 6 y 7 comentaremos otras posibles motivaciones). Estos últimos son conceptos conjuntivos, híbridos. Están constituidos conjuntivamente por un concepto no mental, SER VERDADERO, y otros conceptos. Eso impide - en virtud de (W4) - que sean mentales. SABER es mental, no está constituido conjuntivamente por un concepto no mental, SER VERDADERO, y otros conceptos. Pero Williamson acepta que saber implica verdad. Así, considera que 'saber' (o el concepto SABER, cuando contemplamos los conceptos como entidades representacionales) refiere a su estado —al estado de saber, que metafísicamente implica la verdad de la proposición sabida - de una forma «simple» o «primitiva»; una forma no híbrida, que no combina conceptualmente un factor no mental con otros factores. La idea sería que DOXACERTAR y JUSTACERTAR sí combinan conceptualmente, de forma artificiosa, un factor no mental con otros factores.

Quizá fuera procedente destacar que esa noción de simplicidad (relación referencial primitiva, no híbrida) en la relación semántica entre la representación (sea expresión lingüística o concepto) y el estado representado debería clarificarse más. Es esencial comprenderla para comprender la posición de Williamson. No seguiré dicha línea de crítica, pues voy a utilizar esa misma noción de simplicidad presupuesta por Williamson. Si es un concepto oscuro, entonces será poco clara la hipótesis metafísico-«antropológica» que plantearé a continuación, pero será también poco clara - y por tanto problemática- la concepción de Williamson que pretendo mostrar que es problemática. En definitiva, concerniría principalmente a Williamson despejar posibles dudas sobre la inteligibilidad de la noción de simplicidad invocada.

Consideremos esta hipótesis, a la que —en una primera aproximación- podemos llamar metafísico-«antropológica»:

(H) Existe al menos una comunidad de sujetos cuya lengua dispone de una expresión lingüística, ' $\mathrm{x}$ ', que expresa un determinado concepto, $\mathrm{X}$, los cuales refieren al estado de doxacertar de forma simple; una forma análoga (no híbrida, no artificiosamente combinatoria de un factor mental y otro no mental) a como el verbo 'saber' y el correspondiente concepto SABER refieren al estado de saber ${ }^{6}$

6 En sentido estricto, no sería una hipótesis específicamente antropológica, pues -como indicaré pronto- su ámbito no está restringido a seres humanos. Y ese mayor alcance de la hipótesis habla a fa- 
Focalizaré la discusión en torno a $(\mathrm{H})$, pero todo lo que diré podrá decirse análogamente respecto a una hipótesis semejante sobre justacertar:

$\left(\mathrm{H}^{*}\right)$ Existe al menos una comunidad de sujetos cuya lengua dispone de una expresión lingüística, ' $z$ ', que expresa un determinado concepto, $\mathrm{Z}$, los cuales refieren al estado de justacertar de forma simple; una forma análoga (no híbrida, no artificiosamente combinatoria de un factor mental y otro no mental) a como el verbo 'saber' y el correspondiente concepto SABER refieren al estado de saber

Son convenientes algunas reflexiones para calibrar mejor el significado de $(\mathrm{H})$. Primero una cuestión menor, de orden notacional. En rigor, la expresión ' $x$ ' que aparece en la formulación de $(\mathrm{H})$ no debería ir entre comillas (y cabe decir algo similar respecto a la aparición de ' $\mathrm{X}$ '), pues es una variable (que varía sobre expresiones lingüísticas). Dicho de otro modo, las expresiones lingüísticas tematizadas en $(\mathrm{H})$ no necesariamente están compuestas por una sola letra, que además pertenezca a nuestro alfabeto. Sin embargo, creo que la exposición será más fluida si somos laxos y asumimos, en efecto, que ' $x$ ' es una expresión lingüística (un ejemplo particular del tipo de expresión lingüística) de las cuales trata $(\mathrm{H})$. Así, conforme a las opciones notacionales y terminológicas de la sección 1, diremos que - en el lenguaje usado por los sujetos mencionados por $(\mathrm{H})$ - ' $\mathrm{x}$ ' refiere al estado de doxacertar y expresa el concepto X (correferencial con, pero no idéntico al, concepto DOXACERTAR).

El término 'doxacertar' se introdujo estipulando que significaba lo mismo que 'creer algo verdadero’ (en el sentido explicado en la sección 1). Así, acepto —aunque sólo fuera por mor de la argumentación- que DOXACERTAR es un concepto conjuntivo híbrido, que no denota a su estado con la simplicidad con que supuestamente SABER denota al suyo. Pero los hablantes mencionados en la $(\mathrm{H})$ no habrían hecho, respecto a la expresión 'x', algo similar a dicha estipulación. Habrían «hecho» algo similar a lo que, según Williamson, hemos «hecho» nosotros con la expresión 'saber' (lo que hemos «hecho» las diferentes comunidades lingüísticas que tenemos un verbo traducible como 'saber'). El término ' $\mathrm{x}$ ' y el correspondiente concepto $\mathrm{X}$ se habrían acuñado en su lenguaje análogamente a cómo el término 'saber' y el correspondiente concepto SABER se ha acuñado en nuestros lenguajes: de forma primitiva, simple; de forma tal que aunque el estado denotado por ' $\mathrm{x}$ ' implica verdad, el concepto SER VERDADERO no es uno de los conjuntos componentes del concepto X. Usamos 'saber' y SABER sin haberlos introducido estipulativamente como conceptos conjuntivos híbridos. También los sujetos postulados en (H) usarían ' $\mathrm{x}$ ' y X sin haberlos introducido estipulativamente como conceptos conjuntivos híbridos. Por eso he destacado — en el último párrafo- que dicho concepto X no es idéntico al concepto DOXACERTAR.

La verdad o falsedad de $(\mathrm{H})$ es una cuestión empírica contingente. No tengo ningún indicio para suponer que $(\mathrm{H})$ sea, de hecho, verdadera. En realidad, al menos cuando consideramos $(\mathrm{H})$ restringida a seres de nuestra especie, me parece una hipótesis más probablemente falsa que verdadera. Mi posición, pues, no depende de la supuesta plausibilidad de $(\mathrm{H})$; mi posición sólo requiere la plausibilidad de una tesis muchísimo más débil que $(\mathrm{H})$ :

vor de mi argumentación. (Respecto a que tanto la expresión como el concepto refieren a cierto estado, cf. el segundo párrafo de la sección 1.) 
la tesis de que $(\mathrm{H})$ no es metafisicamente imposible. En efecto, $(\mathrm{H})$ no parece una imposibilidad metafísica. La mera posibilidad de que hubiera usos de lenguajes y conceptos con las características indicadas en $(\mathrm{H})$ tendrá consecuencias — en virtud de (W3), según veremossobre la cuestión de si doxacertar es o no un estado mental (incluso aunque de hecho no existan lenguajes y conceptos de ese tipo; es decir, incluso aunque de hecho $(\mathrm{H})$ sea falsa). En la sección 7 evaluaremos una propuesta específica para sostener que $(\mathrm{H})$ es imposible. Ahora querría añadir algunas breves consideraciones para reforzar la intuición preteórica inicial: que en algunos mundos posibles ocurre lo que $(\mathrm{H})$ establece.

Consideremos el estado de saber. La historia de la evolución humana ha dado lugar a diferentes lenguas naturales que típicamente disponen de expresiones simples cuya referencia es dicho estado. Tales expresiones son 'saber' y sus sinónimos en esas otras lenguas, todas las cuales - asumimos- expresan el mismo concepto: SABER. Consideremos ahora el estado de doxacertar. Imaginemos que nuestra especie hubiera evolucionado de forma diferente, dando lugar a algunas lenguas naturales que típicamente dispusieran de expresiones simples cuya referencia fuera dicho estado. Sería difícil sostener que estamos imaginando algo metafísicamente imposible. Cada mundo posible en que nuestra historia cognitiva y lingüística se ajusta a esa descripción es un mundo en el cual se cumple $(\mathrm{H})$.

En realidad, la impresión de que $(\mathrm{H})$ es posible se ve reforzada al constatar que no es una hipótesis restringida a sujetos de nuestra especie (por ello no es del todo acertada la etiqueta 'antropológica'). $(\mathrm{H})$ pudiera ser verdadera en virtud de historias evolutivas posibles de otras especies biológicas, no necesariamente pertenecientes a nuestro planeta. $\mathrm{Si}(\mathrm{H})$ fuera imposible, entonces el estado de doxacertar no podría ser la referencia de una expresión lingüística simple utilizada por nuestra especie ni por ninguna otra posible especie de sujetos racionales.

Exploremos las consecuencias, en el seno de TWM, de que $(\mathrm{H})$ sea una posibilidad metafísica. Dadas las semejanzas entre el posible concepto $\mathrm{X}$ y el concepto SABER, si este último es mental resulta difícil defender que $\mathrm{X}$ no es un concepto mental. En la sección 6 evaluaremos una propuesta específica para sostener que X no sería un concepto mental. De momento, exploremos las consecuencias de aceptar, también, que $\mathrm{X}$ sería un concepto mental. Así, puede haber un concepto mental del estado de doxacertar. Por consiguiente, según (W3), doxacertar es un estado mental. Pero DOXACERTAR no es un concepto mental. Un posible concepto mental, $X$, es necesariamente coextensional con un concepto no mental, DOXACERTAR. Es sensato descartar la tesis (W5), que aconsejaba desestimar ese tipo de coincidencias por resultar difícilmente explicables. Lo que sería difícilmente explicable es pretender que $(\mathrm{H})$ es imposible, o que $(\mathrm{H})$ es posible pero $\mathrm{X}$ no sería mental aunque lo sea SABER. Finalmente, si descartamos la tesis (W5), entonces están en peligro - según se ha mostrado en las secciones 2 y 3 - las dos hipótesis relevantes de Williamson sobre las posibilidades de definir el conocimiento: su hipótesis negativa (W6) y su definición (D).

\section{Operadores de estados mentales factivos}

En lo referente a la viabilidad de la definición del saber propuesta por Williamson, su posición sigue siendo problemática pero quizá no de forma tan contundente si en lugar de considerar la definición informal, (D), nos fijamos en su definición precisa oficial. Esta otra definición utiliza la noción técnica de operador de estado mental factivo, operador EMF en 
adelante (FMSO es la denominación en inglés empleada por Williamson), y establecería lo siguiente:

$\left(D^{*}\right)$ Saber es el (tipo de) estado mental más general denotado por un operador EMF

Williamson presenta el concepto de operador EMF con algunos casos ilustrativos acompañados de ciertas estipulaciones. En su opinión, 'saber' y 'recordar' son operadores EMF. Por otra parte, estipula que un operador EMF es semánticamente inanalizable entendiendo por tal que no es sinónimo de ninguna expresión compleja cuyo significado se compone del significado de sus partes. ${ }^{7}$

Obviamente, un operador que denota paradigmáticamente el estado de saber y es catalogable — según Williamson- como operador EMF es 'saber'.

Según vimos, Williamson protegería su definición (D) ante presuntos contraejemplos (estados mentales más generales que saber) como doxacertar y justacertar afirmando que éstos no son estados mentales. En términos de la definición $\left(\mathrm{D}^{*}\right)$, los presuntos contraejemplos serían también los estados de doxacertar y justacertar. Los operadores EMF que los denotarían, respectivamente, son las expresiones ' $\mathrm{x}$ ' $\mathrm{y}$ ' $\mathrm{z}$ ' mencionadas en las hipótesis $(\mathrm{H}) \mathrm{y}$ $\left(\mathrm{H}^{*}\right)$. Ahora Williamson tendría, para $\left(\mathrm{D}^{*}\right)$, una defensa adicional: podría proponer, además, que ' $\mathrm{x}$ ' y 'z' no son operadores EMF, no sólo porque denotan propiedades no mentales, sino por no cumplir con la estipulación mencionada respecto a tales operadores: por ser semánticamente analizables. Pero, como contrapartida, su definición $\left(\mathrm{D}^{*}\right)$ tiene un potencial punto débil que no tenía (D): requiere el supuesto de que 'saber' sí sea un operador EFM. Si tales operadores son, por estipulación, semánticamente inanalizables, dicho supuesto ya acarrea consigo algunas de las hipótesis negativas sustantivas de Williamson sobre la definibilidad del conocimiento (aunque no implica, en particular, su tesis (W6)).

En cualquier caso, es controvertido que ' $x$ ' $y$ ' $z$ ' sean sinónimos de 'doxacertar' y 'justacertar' (esa defensa adicional de $\left(D^{*}\right)$ invocaría dicha sinonimia para sostener que ' $x$ ' y ' $z$ ' son semánticamente analizables y, por tanto, no son operadores EMF). Aunque 'X que P' y '(cree que $\mathrm{P}$ ) y ( $\mathrm{P}$ es verdad)' refieren al mismo estado, no es obvio que sean sinónimos. En particular, las comunidades de que habla $(\mathrm{H})$ no habrían estipulado (ni tampoco lo estipulo yo) que ' $\mathrm{X}$ ' designe al estado de doxacertar. ${ }^{8}$ Podría defenderse dicha sinonimia alegando lo siguiente: un epistemólogo usuario del término ' $\mathrm{x}$ ' $\mathrm{y}$ del correspondiente concepto $\mathrm{X}$ quizá estaría en condiciones de constatar - a partir de la reflexión conceptual, no empírica- que la propiedad denotada por ' $x$ ' es la propiedad de tener cierta creencia verdadera. Pero esto conlleva dos inconvenientes. (a) Nos retrotrae al punto mencionado al final del párrafo previo: un epistemólogo usuario del término 'saber' y del correspondiente concepto SABER

7 Cf. Williamson (2009a, 285; 2009b, 364). Aunque en Williamson (2000, 36, 39 y 40) lo da a entender, no era tan claro que se pretendiera incluir estipulativamente en el significado de «operador EMF» dicha «inanalizabilidad» semántica. Así, por ejemplo, Reed $(2005,137)$ asume que no es una estipulación, sino una mera hipótesis. Cf. también Sosa $(2009,206)$.

8 Por esa razón, la propuesta de Reed (2005), sobre «gnows», difiere sustancialmente de la mía. Reed describe cierto tipo de estado específico complejo (mirar a un granero en buenas condiciones de visibilidad, estando, sin saberlo, en un área con muchos graneros falsos; el ejemplo original procede de Goldman) y luego propone: «Introduzcamos un término semánticamente no analizable [gnows] para casos de este tipo» (Reed 2005, 138). Williamson sostendría - contra Reed- que nos está vedada dicha estipulación; el término «gnows», introducido de esa forma estipulativa, es semánticamente analizable. 
quizá estaría en condiciones de constatar — a partir de la reflexión conceptual— que saber es la propiedad de tener cierta creencia verdadera justificada cuando concurre algún otro factor adicional que ese epistemólogo hubiera logrado identificar. Willianson asume que está condenada al fracaso la tarea de ese epistemólogo; a su juicio, no existe ese factor adicional como elemento individualmente aislable. Al clasificar 'saber' como operador EMF, Williamson también está asumiendo la imposibilidad de ese logro; aunque el siguiente punto, (b), es asimismo relevante en esa clasificación. (b) Generaría tensiones en la metafilosofía de Williamson que una constatación teorética derivada de (su versión de en qué consiste) la reflexión conceptual pudiera basar una afirmación de sinonimia semántica.

En resumidas cuentas, aunque 'doxacertar' y 'justacertar' sean términos semánticamente analizables (en el sentido que se ha indicado), Williamson tendría dificultades para justificar que también ' $\mathrm{x}$ ' $\mathrm{y}$ ' $\mathrm{z}$ ' son semánticamente analizables sin serlo 'saber'. Por tanto, tendría dificultades para justificar que no son operadores EMF. Si 'x' y 'z' son operadores EMF, entonces doxacertar y justacertar son contraejemplos también a su definición precisa $\left(\mathrm{D}^{*}\right)$.

\section{Explicaciones de la conducta y el supuesto umbral de lo mental}

En la sección 4 he argumentado que cierta tesis de TWM, la proposición (W5), parece incorrecta. Dicha tesis desempeña un papel crucial para sostener otras cuatro propuestas de Williamson, las cuales - por consiguiente- quedan sin apropiada justificación, salvo en lo relativo a la presunta justificación meramente inductiva de (W6): doxacertar no es un estado mental; justacertar no es un estado mental; la hipótesis negativa (W6); la definición (D). En esa argumentación contra la proposición (W5), he invocado el carácter mental de los conceptos posibles $\mathrm{X}$ y Z, tematizados - respectivamente- por sendas hipótesis que son metafísicamente posibles: $(\mathrm{H})$ y $\left(\mathrm{H}^{*}\right)$. Vamos a considerar dos opciones que le permitirían a Williamson contestar a esa línea de crítica:

(O1) Afirmar que los posibles conceptos $\mathrm{X}$ y $\mathrm{Z}$ no serían conceptos mentales.

(O2) Afirmar que las hipótesis $(\mathrm{H})$ y $\left(\mathrm{H}^{*}\right)$ son metafísicamente imposibles.

En esta sección examinaremos una motivación a favor de (O1). En la sección 7 comentaré brevemente dos motivaciones que podrían sustentar $(\mathrm{O} 2)$. Mi contrarréplica principal, en ambas secciones, será similar: esas motivaciones no son claramente incorrectas, pero introducirían — si Williamson estuviera dispuesto a recurrir a ellas - cierta dosis de arbitrariedad en TWM. En la sección 8 discuto y rechazo otro tipo de motivación a favor de $(\mathrm{O} 2)$.

Según Williamson (2000, sección 2.4), en algunas explicaciones de la conducta el papel desempeñado por las atribuciones de conocimiento es más primordial o básico que los papeles desempeñados por las atribuciones de creencia o de doxacierto. Para ilustrar el punto, Williamson imagina, entre otros, cierto caso, identifica un determinado explanandum y argumenta - convincentemente- que dicho explanandum queda mejor explicado invocando el estado de saber que si invocáramos estados como creer o doxacertar (o justacertar, podríamos también añadir).

J. Comesaña y M. García-Carpintero me han sugerido que esas consideraciones permitirían apoyar la opción (O1). La conexión entre ambos asuntos es indirecta, y Williamson no la propone de forma explícita. Pero creo que la sugerencia es sensata y podría formularse 
en los siguientes términos. Un criterio fundamental para delimitar qué entendemos por mental es la explicación de la conducta. Si hay casos como los descritos por Williamson (en los cuales tenemos mayor poder explicativo - de la conducta de un agente- utilizando el concepto SABER que utilizando CREER o DOXACERTAR) y no hay casos -o son significativamente menos frecuentes - en que suceda lo contrario (atribuir saber supone perder poder explicativo frente a atribuciones de creencia o de doxacierto), entonces eso indica que SABER es un concepto mental en mayor grado que el grado en que pudieran ser mentales CREER y DOXACERTAR (y JUSTACERTAR), si es que éstos son también conceptos mentales. ${ }^{9}$ Puesto de otro modo: cuando exploramos formas variadas de conducta racional, estamos inclinados a aceptar (W7):

(W7) Usar el concepto SABER permite, en términos generales, explicar mejor la conducta que usar el concepto CREER o el concepto DOXACERTAR (o el concepto JUSTACERTAR)

Y una vez aceptamos (W7), debemos aceptar (W8):

(W8) SABER es un concepto mental en mayor grado que CREER y DOXACERTAR (y JUSTACERTAR), si es que éstos son también conceptos mentales.

Ésa es -me parece- la idea sugerida por Comesaña y García-Carpintero.

Sin embargo, (W7) es una proposición controvertida, y la transición desde (W8) hasta la opción $(\mathrm{O} 1)$ requeriría otros dos pasos adicionales, también controvertidos. Respecto a (W7), Williamson parece considerar, en efecto, que no podríamos identificar muchas situaciones en las cuales se inviertan los términos respecto a la capacidad explicativa de unos y otros conceptos; por lo general, SABER permite explicar mejor las conductas que CREER o DOXACERTAR. Pero no ofrece mayores indicios de ello. Quedaría abierta la posibilidad de identificar otro casos (o los mismos casos, pero proponiendo explananda distintos a los sugeridos por Williamson) en los cuales quizá al usar los conceptos CREER o DOXACERTAR se gana poder explicativo frente al uso de SABER. No obstante, eso serían meras especulaciones. Concedamos que (W7) es verdadera.

Supongamos que aceptamos también la inferencia (no deductiva) de (W7) a (W8). ¿Cómo se justificaría la opción (O1), negar el carácter mental de los conceptos $\mathrm{X}$ y Z - mencionados en las hipótesis $(\mathrm{H})$ y $\left(\mathrm{H}^{*}\right)$ - a partir de (W8)? Esa transición es indirecta y conlleva otras dos complicaciones. (W7) y (W8) conciernen - entre otros- a los conceptos DOXACERTAR y JUSTACERTAR. Pero nuestra discusión es sobre los posibles conceptos (correferenciales con ellos, pero no idénticos) X y Z. En particular, la verdad de (W7) no garantiza la verdad de esta otra tesis, (W7*), que sería la proposición relevante:

$\left(\mathrm{WT}^{*}\right)$ Usar el concepto SABER permite, en términos generales, explicar mejor la conducta que usar el concepto X (correferencial con el concepto DOXACERTAR) o el concepto Z (correferencial con el concepto JUSTACERTAR)

9 En una interpretación alternativa, las condiciones mencionadas no serían indicio de que SABER es un concepto mental en mayor grado que otros, sino de que el carácter mental de SABER (quizá no gradual) está mejor establecido, o es menos dudoso, que el carácter mental de esos otros conceptos. La diferencia no afecta al fondo del asunto; aunque requeriría modificar correlativamente la tesis (W8) y otras subsiguientes. 
No creo que tengamos intuiciones suficientemente nítidas sobre el contraste (respecto a la explicación de la conducta) entre el concepto SABER y esos otros conceptos: X y Z. Supongamos que los sujetos mencionados en las hipótesis $(\mathrm{H})$ y $\left(\mathrm{H}^{*}\right)$ - o algunos de ellostuvieran también otro término simple, 's', traducible por 'saber'. Podemos asumir que ese concepto $S$ sería, a grandes rasgos, el concepto SABER. Cuando tales sujetos elaborasen explicaciones de la conducta, ¿serían también, por lo general, mejores sus explicaciones si usaran el concepto $S$ que si usaran los conceptos $\mathrm{X}$ o Z ? La hipótesis $\left(\mathrm{W} 7^{*}\right.$ ) responde afirmativamente; pero no es una cuestión trivial, ni siquiera una vez aceptada (W7).

De todos modos, supongamos - por mor de la argumentación- que aceptamos $\left(\mathrm{W} 7^{*}\right)$, y - de nuevo- aceptamos también la inferencia (no deductiva) de (W7*) a (W8*), que sería la proposición relevante análoga a (W8):

(W8*) SABER es un concepto mental en mayor grado que $\mathrm{X}$ y $\mathrm{Z}$, si es que éstos son también conceptos mentales.

Llegamos ahora a la segunda complicación, de mayor importancia. Como resulta obvio, $\left(\mathrm{W}^{*}\right)$ no confiere apoyo — por sí misma - a la idea de que X y Z no son conceptos mentales. Sólo le presta apoyo si va acompañado de ciertas hipótesis auxiliares. Éstas podrían formularse así: Un criterio fundamental para delimitar qué entendemos por mental es la explicación de la conducta. En algunas explicaciones se usan conceptos como SABER y CREER. En otras, se usan conceptos como $X$ y $Z$. No sólo son éstas últimas - por lo general-explicativamente menos adecuadas que las primeras, sino que no llegan al umbral minimo exigible para poder considerar que tales conceptos son, en efecto, mentales. Williamson contrasta usos de SABER con usos de CREER y DOXACERTAR, según se indica en (W7). Pero acepta que CREER es un concepto mental. Por tanto, en la explicación de la conducta el concepto CREER satisface esos requisitos mínimos para ser clasificado como mental. Pero - si Williamson quisiera usar sus consideraciones a favor de (W7) para apoyar la opción (O1), conforme a la sugerencia de Comesaña y García-Carpintero- necesita ese tipo de hipótesis auxiliares adicionales, cuyo punto principal viene a ser esto: la línea de demarcación (de lo mental) deja a SABER y CREER a un lado, y deja a $\mathrm{X}$ y $\mathrm{Z}$ al otro lado. ¿Por qué hemos de suponer que ha de ser ésa la posición de dicha línea de demarcación? Si nos basamos en los comentarios de Williamson (2000, sección 2.4) respecto a las explicaciones de la conducta, también podríamos dudar de que CREER sea mental. Estrictamente, esos comentarios sólo resaltan que SABER es más prioritario que CREER y que DOXACERTAR, no que éstos no alcancen cierto umbral mínimo, ni que CREER sí alcance ese umbral pero no así DOXACERTAR. En vista de todo ello, creo que sería un poco arbitrario sostener que la línea de demarcación está situada precisamente en esa posición.

\section{Sobre la naturalidad y elegibilidad de los estados}

Un objetor podría intentar seguir la opción (O2) invocando cierta hipótesis empírica restrictiva: Si la lengua natural L tiene una expresión simple que refiere al estado de creer y tiene una expresión simple que refiere al estado de saber, es muy improbable que L también tenga una expresión simple que refiera al estado de doxacertar. Sin embargo, aunque esa hipótesis fuera cierta, no le sirve al objetor, pues $(\mathrm{O} 2)$ requiere la imposibilidad de $(\mathrm{H})$, no meramente su baja probabilidad en el espacio lógico. Además, $(\mathrm{H})$ no implica que los usuarios de 
la expresión lingüística ' $\mathrm{x}$ ' usen también expresiones lingüísticas que expresen los conceptos CREER y SABER.

Una defensa de (O2) emparentada con esa me ha sido propuesta por M. García-Carpintero. Sostiene que estados como doxacertar y justacertar no serían suficientemente naturales o simples como para resultar «elegibles» mediante una única expresión lingüística o mediante un concepto no híbrido. El estado de saber, por el contrario, sí es natural y puede ser referido de forma primitiva por el término 'saber' y por el concepto SABER. ${ }^{10}$ Sin pretender que el grado de artificiosidad sea el mismo, los términos 'doxacertar' y 'justacertar' serían —en ese sentido- análogos a 'verzul'. Goodman (1955) introdujo el concepto para diseñar un enigma cercano al problema de Hume sobre la inducción, asignando a 'verzul' [grue, en el original] este significado (que actualizo sin modificarlo en lo principal): $x$ es verzul si y sólo si ( $x$ es un objeto verde examinado antes del año 3.000 o es un objeto azul no examinado antes del año 3.000). Tenemos términos y conceptos relativamente simples para denotar la propiedad de ser verde ('verde' y VERDE, respectivamente); lo mismo respecto a ser azul. Pero no los tenemos para denotar la propiedad de ser verzul. No es casual: el carácter poco natural o simple de la propiedad verzul (en un sentido parcialmente técnico de natural y simple, cercano al tematizado por Lewis y otros autores) imposibilitaría ser denotada por un término o un concepto primitivo. Ciertas teorías ontológicas incluso negarían que verzul fuera una propiedad. También 'doxacertar' se define artificiosamente recurriando a los conceptos simples (o comparativamente simples) de CREER y SER VERDADERO. Por eso, el estado denotado no tendría la naturalidad o simplicidad suficiente para ser la referencia de un término primitivo, ' $x$ '. Debido ello, $(\mathrm{H})$ sería una hipótesis imposible.

Presento dos puntualizaciones, a modo de réplica. Primero, doxacertar es un estado considerablemente menos artificioso que ser verzul. Recordemos, incluso, que existe ya un verbo simple, 'acertar' (y sus sinónimos en otras lenguas), que — aunque tiene un sentido literal más general- cuando el contexto permite entender que se está hablando sobre las operaciones cognitivas de un sujeto, puede usarse precisamente para referirse a doxacertar. ¿Tan extraño sería que pudiera haber una expresión simple que refiriese a ese sentido restringido de acertar? Insisto, de nuevo: mi posición no depende de que exista de hecho esa expresión simple en algún lenguaje; depende de que no sea metafísicamente imposible que exista.

En segundo lugar, mi réplica central es similar a lo indicado en la sección anterior sobre la sugerencia para sostener $(\mathrm{O} 1)$. Análogamente a lo dicho allí, no basta con aceptar que creer y saber son más naturales, menos artificiosos, que doxacertar y justacertar. ¿Tenemos alguna justificación (no arbitraria, no ad hoc) para suponer que los estados de doxacertar y justacertar no alcanzan el nivel mínimo de naturalidad requerido para ser representable mediante términos y conceptos primitivos? Volvamos a la comparación con verzul. Estamos bastante inclinados a aceptar que ninguna posible historia evolutiva de nuestra especie pudiera haber sido tan diferente a la historia real como para que los humanos hubieran acuñado términos y conceptos primitivos que denotaran ser verzul. Aunque con reparos, la

10 Lewis (1983) es un artículo clásico en el cual se tematiza esa idea de naturalidad de las propiedades como requisito para que éstas puedan ser representadas, o «elegidas», como referentes de expresiones o conceptos semánticamente primitivos. Me parece una idea plausible, y he intentado aplicarla en otros trabajos para afrontar ciertos problemas filosóficos; cf., por ejemplo, Pérez Otero (1999, cap. 2; 2016a, sección 6). 
inclinación también está presente cuando no nos restringimos a seres humanos: ¿cómo registrarían o detectarían — de forma simple- esa «propiedad» los sujetos relevantes? Sin embargo (y asumiendo que 'creer' y 'saber' son expresiones primitivas) no se nos antojan igualmente inverosímiles historias evolutivas alternativas de nuestra especie, o historias evolutivas de otras especies, que dieran lugar a términos y conceptos primitivos que denotaran doxacertar o justacertar.

En cualquier caso, debemos tener claro el coste teórico derivado de proponer la imposibilidad de las hipótesis $(\mathrm{H})$ y $\left(\mathrm{H}^{*}\right)$ basándonos en esa propuesta. Hay una separación de lo natural frente a lo artificioso (eso me parece aceptable); esa separación dejaría a un lado estados como creer y saber, y dejaría en el lado opuesto estados como doxacertar y justacertar, los cuales no son suficientemente naturales como para resultar «elegibles» por conceptos primitivos. Postular que la línea de demarcación se sitúa en esa zona, precisamente, permitiría evitar mi objeción contra la teoría de Williamson; pero acarrearía — a mi juicio- cierto grado de arbitrariedad. ${ }^{11}$

\section{Condiciones de aplicación de los conceptos}

En relación con la tesis crucial discutida en la sección previa (la tesis de que $(\mathrm{H})$ es metafísicamente posible), un/a anónimo/a evaluador/a de Theoria señala dos puntos: (a) la carga de la prueba respecto a si $(\mathrm{H})$ es o no metafísicamente posible recae en quien pretende (como es mi caso) que $(\mathrm{H})$ es posible, no en quien pretende que $(\mathrm{H})$ es imposible; (b) mostrar que $(\mathrm{H})$ es metafísicamente posible requeriría mostrar que el concepto X es lógico-conceptualmente posible, lo cual requeriría - a su vez- indicar cómo se usa, en el sentido de especificar cuáles son sus condiciones de aplicación.

Respecto a (a), es muy difícil determinar con exactitud qué proporción de carga de la prueba corresponde a cada parte en el debate. Pero, basándome en las consideraciones que he presentado (en las secciones 4 y 7 ), me parece razonable sostener que una parte significativa de dicha carga recae en el contrincante, el filósofo que apoya la opción O2. En términos generales (sin negar que haya excepciones), me parece prudente aceptar la siguiente reflexión metodológica. Supongamos que $\mathrm{G}$ es una hipótesis que afirma la existencia de ciertos tipos de hechos y $G$ no parece imposible. Siendo así, si nos preguntamos sobre el valor veritativo de $G$, esperamos que su defensor aporte indicios en favor de $G$; pero si nos preguntamos sobre si $G$ es o no posible, esperamos que quien aporte indicios en favor de su posición sea principalmente quien afirma la imposibilidad de $\mathrm{G}$ (porque las afirmaciones de imposibilidad tienen, típicamente, un contenido informacional muy fuerte; en general, la mera apariencia de que algo es posible constituye indicio de que es posible).

${ }^{11}$ Sería erróneo suponer que las reflexiones contenidas en el cap. 3 de Williamson (2000) sobre el contraste entre condiciones primas [prime] y compuestas [composite] pudieran usarse en favor de las opciones $(\mathrm{O} 1)$ u $(\mathrm{O} 2)$. Allí ofrece Williamson una demostración de que saber es un estado primo (es decir, no compuesto, en un cierto sentido técnico que define previamente). Pero combate a un opositor internista cuyas concepciones de doxacertar y justacertar implicaran que ésos son estados compuestos. Conforme al externismo, también doxacertar y justacertar son estados primos; por tanto, para un externista ningún contraste entre ellos y saber derivaría de esa discusión. 
No obstante, diré también algo respecto a (b), pues ese/a evaluador/a - y tal vez otros lectores- quizá consideren que $(\mathrm{H})$ parece imposible. Un paso importante en la dirección de especificar las condiciones de aplicación de un concepto consiste en especificar las condiciones de verdad de las oraciones simples que involucran (términos que expresan) ese concepto. Cualquiera concederá que para el caso del concepto $\mathrm{X}$ esa tarea es bien sencilla: cuando un usuario del concepto $\mathrm{X}$ afirme, empleando el término ' $\mathrm{x}$ ', que $S$ está en la relación expresada por ' $x$ ' con cierta proposición, su afirmación será verdadera si y sólo si $S$ cree dicha proposición y ésta es verdadera. Entiendo que tales condiciones de verdad no agotan las condiciones de aplicación de X. Cabe pensar que la noción de condiciones de aplicación permite individualizar los conceptos; las condiciones de verdad mencionadas no individualizan el concepto X, pues son compartidas por el concepto DOXACERTAR. Pero, al menos en lo tocante a las condiciones de verdad de $\mathrm{X}$, no deberíamos dudar sobre la inteligibilidad o la posibilidad lógico-conceptual de X (salvo que dudemos sobre la inteligibilidad o la posibilidad lógico-conceptual de DOXACERTAR).

El punto crucial es la parte restante de sus condiciones de aplicación (la parte no compartida por DOXACERTAR). ¿En qué consistiría aplicar el concepto X? Reiteraré la anología ya resaltada entre X y SABER, y añadiré otra consideración. Usar X requiere aplicar $\mathrm{X}$ conforme a esas condiciones de verdad (naturalmente, con posibilidad de aplicarlo de forma errónea, como pasa con todos los conceptos categoremáticos); y aplicarlo al estado de doxacertar con el mismo tipo de simplicidad con que - según Williamson - aplicamos SABER al estado de saber. En particular, es factible identificar algunos rasgos indicativos de que un sujeto está aplicando-usando X y no está aplicando-usando DOXACERTAR (y viceversa). Aunque casi todos aceptamos que SABER implica creencia verdadera, dicha implicación no es una absoluta trivialidad. Podemos imaginar la tarea de elucidación conceptual emprendida por un filósofo que - siglos atrás - nos invita a aceptar dicha implicación; que esa elucidación no es trivial queda atestiguado por el hecho de que algunos epistemólogos han postulado casos de saber sin creencia, e incluso de saber no factivo. Dada nuestra introducción estipulativa del término 'doxacertar', proponer que DOXACERTAR implica creencia verdadera sí sería una completa trivialidad; trivialidad potencialmente contrastable en las reacciones de usuarios del concepto DOXACERTAR a quienes se les propusiera dicha «elucidación». Pues bien, tenemos aquí un elemento que distingue la posible aplicación del concepto DOXACERTAR de la posible aplicación del concepto $\mathrm{X}$ : las reacciones de usuarios de $\mathrm{X}$ ante una elucidación de ese concepto según la cual se les indique que $\mathrm{X}$ implica creencia verdadera serán más semejantes a las de usuarios de SABER ante la correspondiente elucidación que a las de usuarios de DOXACERTAR; incluso podemos imaginar usuarios de $\mathrm{X}$ reticentes ante esa elucidación (que postulen contraejemplos, como algunos postulan contraejemplos a que SABER implique creencia verdadera).

En definitiva, la doble tarea de identificar con precisión las condiciones de verdad relevantes y especificar rasgos que distinguirían aplicar-usar el concepto DOXACERTAR de aplicar-usar el concepto X (combinada con la analogía entre la simplicidad de SABER y la simplicidad de $\mathrm{X}$ ) nos aproximan suficientemente a la comprensión de las condiciones de aplicación de $\mathrm{X}$ como para aceptar - a mi juicio- que $\mathrm{X}$ es un concepto lógico-conceptualmente posible, o que - cuando menos - quien niegue su posibilidad lógico-conceptual debería precisar en qué basa sus dudas. 


\title{
REFERENCIAS
}

Gettier, Edmund. 1963. Is Justified True Belief Knowledge?. Analysis 23: 121-123.

Goodman, Nelson. 1955. The New Riddle of Induction. Capítulo II de N. Goodman, Fact, Fiction, and Forecast. Cambridge Mass.: Harvard University Press.

Greenough, Patrick y Pritchard, Duncan (eds.). 2009. Williamson on Knowledge. Oxford: Oxford University Press.

Lewis, David. 1983. New Work For a Theory of Universals. Australasian Journal of Philosophy 61: 343-377.

Pérez Otero, Manuel. 1999. Conceptos modales e identidad. Barcelona: Edicions Universitat de Barcelona.

Pérez Otero, Manuel. 2016a. Respuestas disposicionalistas al problema wittgensteiniano-kripkeano sobre el significado y el seguimiento de reglas. Crítica. Revista Hispanoamericana de Filosofía 48/143: 21-45.

Pérez Otero, Manuel. 2016b. La teoría de Williamson sobre los conceptos y los estados mentales. En Proceedings of the Eighth Meeting of the SEFA, 148-151. Oviedo: KRK Ediciones.

Pérez Otero, Manuel. 2017. Teorías de la referencia, filosofía experimental y calibración de intuiciones. THEORIA: An International Journal for Theory, History and Foundations of Science 32/1: 41-62.

Reed, Baron. 2005. Accidentally Factive Mental States. Philosophy and Phenomenological Research 71: 134142.

Sosa, Ernest. 2009. Timothy Williamson’s Knowledge and its Limits. En P. Greenough y D. Pritchard, eds., 203-216.

Sosa, Ernest. 2015. Judgment and Agency. Oxford: Oxford University Press.

Williamson, Timothy. 2000. Knowledge and its Limits. Oxford: Oxford University Press.

Williamson, Timothy. 2009a. Reply to Quassim Cassam. En P. Greenough y D. Pritchard, eds., 285-292.

Williamson, Timothy. 2009b. Reply to Ernest Sosa. En P. Greenough y D. Pritchard, eds., 364-369.

\begin{abstract}
Manuel Pérez Otero (Doctorado en Filosofía, 1996) es Profesor Titular en el Departamento de Filosofía de la Univ. de Barcelona, y miembro de LOGOS (Research Group in Analytic Philosophy) desde su fundación. Investiga preferentemente en epistemología, filosofía del lenguaje, lógica filosófica y metafísica. Sobre esas temáticas ha publicado varios libros y artículos en revistas especializadas como Crítica, Dialéctica, Disputatio, European Review of Philosophy, Grazer Philosophische Studien, History and Philosophy of Logic, Pacific Philosophical Quarterly, Philosophical Studies, Philosophical Papers, Philosophy and Phenomenological Research, Synthese y Teorema.
\end{abstract}

Dirección: Departamento de Filosofía. Facultad de Filosofía. Univ. de Barcelona. / LOGOS. Calle Montalegre, 6. 08001 Barcelona. E-mail: perez.otero@ub.edu 